BEST PRACTICE

\title{
Management of inflammatory bowel disease
}

\author{
M Nayar, J M Rhodes
}

Postgrad Med J 2004;80:206-213. doi: 10.1136/pgmj.2003.013722

Ulcerative colitis and Crohn's disease result from an interaction between genetic and environmental factors. Only one gene, NOD2/CARD15, has been clearly identified; a minority of people with alteration of this gene develop Crohn's disease. The NOD2/CARD15 protein is thought to be involved in defence against intracellular bacteria. This supports the idea that Crohn's disease and ulcerative colitis result from altered immunological responses to the normal intestinal flora. Life expectancy is normal in ulcerative colitis and nearly so in Crohn's disease, but both conditions cause considerable morbidity. Approximately $80 \%$ of patients with Crohn's disease eventually require surgery, and about $25 \%$ of patients with ulcerative colitis require colectomy. Treatment of ulcerative colitis is generally by corticosteroids for acute disease and mesalazine for maintenance, but the range of therapies for Crohn's disease is expanding. Alternative therapies include immunosuppressives, enteral nutrition, antibiotics, anti-TNF antibody (infliximab), corticosteroids, and surgery. High dosages of corticosteroids may provide symptomatic relief in Crohn's disease but do not affect the long term natural history of the disease, and management strategies should avoid using steroids whenever possible.

See end of article for authors' affiliations

Correspondence to: Professor Jonathan $M$ Rhodes, Department of Medicine, University of Liverpool, Liverpool $\mathrm{L69}$ 3GA, UK; rhodesim@ liverpool.ac.uk

Received 18 August 2003 Accepted 8 October 2003
S ince we do not fully understand the pathogenesis of either ulcerative colitis or Crohn's disease, diagnostic criteria for these conditions have evolved by consensus and dogma over many years. In their most typical clinical forms-for example, stricturing terminal ileal or ileocaecal Crohn's disease and continuous distal ulcerative colitis-there is little argument that the conditions are separate in terms of their genetics, predisposing environmental factors, and response to treatment. In about one third of cases, however, the boundaries are blurred, often necessitating a diagnostic label of "indeterminate colitis". We now know that the NOD2/ CARD15 genetic abnormality is not associated with isolated colonic Crohn's disease, and it is very likely that "typical" ulcerative colitis and isolated colonic Crohn's disease are extreme forms of a phenotypic spectrum rather than completely distinct conditions. It may still be useful to identify where on this spectrum the patient lies because there are some differences in response to treatment. Isolated colonic Crohn's disease, for example, may respond to antibiotics such as metronidazole whereas ulcerative colitis does not, and pouch surgery is more likely to be followed by pouchitis if the preoperative colon conformed to the Crohn's disease phenotype.

\section{GUIDELINES}

The British Society of Gastroenterology published guidelines for the initial biopsy diagnosis of suspected idiopathic inflammatory bowel disease (IBD) in $1997^{1}$ and for the detection and prevention of osteoporosis in IBD in $2000 .^{2}$ Brief guidelines on IBD were published in $1996,{ }^{3}$ but more detailed and updated guidelines are nearing completion.

\section{DIAGNOSIS OF IBD}

Ulcerative colitis typically presents with rectal bleeding or bloody diarrhoea. The differential diagnosis includes infective diarrhoeas due to organisms such as Salmonella, Campylobacter or Shigella spp, amoebic dysentery (if there has been recent travel to the tropics), and carcinoma of the colorectum. Diagnosis therefore requires, as a minimum, stool culture and some form of sigmoidoscopy or colonoscopy. Up to $50 \%$ of relapses of ulcerative colitis are associated with pathogens so stool culture should be performed even in a relapse of established ulcerative colitis.

Crohn's disease may present with almost any gastroenterological symptom depending on the site of the disease. The commonest form of the disease is terminal ileal or ileocaecal disease. This usually presents with chronic colicky abdominal pain and diarrhoea and may be difficult to distinguish from irritable bowel syndrome on history alone. More severe disease will be associated with night sweats and weight loss, and a mass may be present in the right iliac fossa; presentation may be more acute and may mimic acute appendicitis. Serum $\mathrm{C}$ reactive protein concentration is raised in over $95 \%$ of patients with symptomatic Crohn's disease and is useful in distinguishing it from irritable bowel syndrome.

\section{Radiology in IBD}

In an acute attack of ulcerative colitis, a plain abdominal radiograph is useful, not only to exclude toxic megacolon (with a transverse colon dilated to more than $5 \mathrm{~cm}$ in diameter) but also to give an approximate estimate of the extent of disease since an ulcerated colon usually contains no solid faeces. The results of double contrast barium enema can vary from a fine granular

Abbreviations: ASA, aminosalicylate; IBD, inflammatory bowel disease; MRI, magnetic resonance imaging; pANCA, perinuclear antinuclear cytoplasmic antibody; TNF, tumour necrosis factor 
appearance of the bowel wall to diffuse ulceration, thumbprinting (due to mucosal oedema), and narrowing and shortening of the bowel, depending on the severity of the disease. In Crohn's disease of the colon radiographic findings can vary from aphthous ulcers to mural thickening, pseudodiverticula, and the classical cobblestone appearance that results from deep transverse and longitudinal ulcers separated by residual areas of oedematous mucosa. It is commonly associated with skip lesions, stricturing, and relative rectal sparing. ${ }^{45}$ In advanced stages Crohn's disease may lead to the development of fissures, sinus tracts, fistulas, and abscesses. In longstanding ulcerative colitis or Crohn's disease, patients may develop heaped up areas of granulation tissue, known as postinflammatory or "pseudo" polyps. Small bowel barium evaluation is still the mainstay of diagnosis for small intestinal Crohn's disease, although high performance computed tomography and magnetic resonance imaging (MRI) are increasingly used as alternatives. Computed tomography in Crohn's disease is used to diagnose complications such as intra-abdominal abscesses and complex fistulae. ${ }^{6}$ MRI is particularly effective for assessing complex perineal Crohn's disease. In patients who cannot be examined by barium radiology, leucocyte scanning can be performed, in which the patient's own peripheral blood neutrophils are labelled with a radioactive isotope, usually technetium (99m), and reinfused prior to gamma scanning of the abdomen. This is helpful in defining sites of active disease relatively non-invasively but cannot give detailed information about stricturing or ulceration.

\section{Endoscopy in IBD}

Endoscopy with mucosal biopsy is essential for the diagnosis of colitis. Although the typical features of colonic Crohn's disease and ulcerative colitis are somewhat different (table 1), there is considerable overlap.

Intubation and biopsy of the terminal ileum should be possible in most patients with IBD. The severity of IBD as assessed by endoscopy does not correlate reliably with prognosis or predict the clinical response to therapy. ${ }^{7}$ Flexible sigmoidoscopy or careful colonoscopy with enema preparation may, however, be helpful in evaluating ulcerative colitis that is not responding well to treatment in order to consider the need for surgery.

Although gastroscopy will provide a diagnosis of Crohn's disease in less than $5 \%$ of patients, histological gastritis that is not related to Helicobacter pylori can be found in over $50 \%$ of cases. ${ }^{8}$ Push enteroscopy may be very helpful in defining distal duodenal or proximal jejunal disease. The recently developed video capsule endoscopy is likely to be of limited use because of the danger of impaction behind strictures.

\section{Pathology in IBD}

There is considerable overlap in histological features between ulcerative colitis, Crohn's disease, and infectious colitis, so

Table 1 Endoscopic features of Crohn's disease and ulcerative colitis

\begin{tabular}{ll}
\hline Crohn's disease & Ulcerative colitis \\
\hline Rectum often spared & Rectum involved \\
Skip areas & Continuous uniform involvement \\
Aphthous ulcers & Loss of vascular marking \\
Cobblestone appearance & Diffuse erythema \\
Linear or serpiginous ulcers & Mucosal granularity \\
Fistulas & Fistulas rarely seen \\
Ulceration of terminal ileum & Normal terminal ileum (or mild \\
& "backwash" ileitis in pancolitis) \\
\hline
\end{tabular}

the diagnosis should be based on the combination of history, endoscopic and radiological appearances, histology, and microbiology rather than on any single modality. IBD may be distinguished from acute infective colitis by the presence of crypt architectural changes such as crypt branching or sparsity. These features take several weeks to develop and are not likely to be present in an infectious colitis. Crohn's disease is distinguished from ulcerative colitis by inflammation that extends deep to the muscularis mucosae, the presence of granulomata (in about $70 \%$ of cases), and a relative lack of depletion of goblet cell mucus (table 2 ). ${ }^{9}$

Approximately $60 \%$ of patients with ulcerative colitis have a circulating autoantibody-perinuclear antinuclear cytoplasmic antibody (pANCA) - and about $60 \%$ of patients with Crohn's disease have circulating antibodies against an oligomannan present in baker's yeast (anti-Saccharomyces cerevisiae antibody). ${ }^{10}$ Both these antibodies provide intriguing clues to pathogenetic mechanisms, but neither is sufficiently sensitive or specific for routine diagnostic use.

\section{PATHOGENESIS}

Management should ideally be targeted at the underlying causes of the disease. Although neither the pathogenesis of Crohn's disease nor that of ulcerative colitis is clearly understood, a picture is beginning to emerge. Crohn's disease is associated with genetic disorders such as NOD2/CARD15 mutations and with the rarer conditions chronic granulomatous disease and glycogen storage disease type 1B, which involve an inability to kill phagocytosed bacteria, particularly within macrophages. Exacerbation of Crohn's disease by smoking could reflect the known suppressor effect of smoking on macrophage function. Ulcerative colitis is associated with autoantibodies, including pANCA and various antiepithelial antibodies, and with an HLA class 2 allele, DRB1*0103, and seems more likely than Crohn's disease to be an autoimmune disease that is triggered by an inflammatory response to the colonic flora. Its association with non-smoking is poorly understood but might reflect an effect on the brain-gut axis that dampens the proinflammatory effect of stress, something that many patients notice as a factor determining relapse. An alternative explanation could be the marked anti-inflammatory effect of low levels of exposure to carbon monoxide. ${ }^{11}$

\section{TREATMENT OF IBD}

Ulcerative colitis

The choice of appropriate therapy depends on the extent and severity of disease.

\section{Proctitis}

Proctitis-disease limited to the rectum-affects approximately $30 \%$ of patients at presentation, although later spread to more proximal areas can occur. The mainstay of therapy is the topical administration of 5-aminosalicylate (mesalazine or 5-ASA) in the form of suppositories, foams, or enemas. Topical 5-ASA preparations are usually more effective than

Table 2 Pathological changes associated with Crohn's disease and ulcerative colitis

\begin{tabular}{ll}
\hline Crohn's disease & Ulcerative colitis \\
\hline Rectal sparing and skip lesions & Continuous distal disease \\
Mucin retention & Mucin depletion \\
Histiocytic aggregation & Basal plasmacytosis \\
Focal mucosal injury & Diffuse mucosal atrophy \\
Sarcoid-like granulomata & No granulomata \\
Anal involvement & Anal sparing \\
\hline
\end{tabular}


topical corticosteroids. ${ }^{12}$ Limited distal disease, which may be accompanied by rectal bleeding and urgency but not by diarrhoea, will often respond to treatment by suppository, while mild diarrhoea suggests more proximal disease, which will usually require enema or rectal foam. In recurrent proctitis, mesalazine suppositories can be used as a maintenance therapy. When conventional steroids, such as prednisolone or hydrocortisone, are used rectally, approximately half the dose is absorbed and can give rise to significant steroid side effects if used long term. Some of the newer steroids, such as budesonide and prednisolone metasulphobenzoate, are available as enemas and have reduced systemic effects, which justify their use if treatment is prolonged..$^{13}$ In patients who cannot tolerate topical therapy, oral mesalazine can be used but is probably less effective. Occasionally proctitis can fail to settle with these measures. This can be a very difficult and frustrating problem and may even lead to the need for total colectomy (in about $5 \%$ of cases) because of impaired quality of life. A partial colectomy is not appropriate as the colitis almost always promptly recurs more proximally. Short courses of systemic corticosteroids backed up by maintenance azathioprine, as for more extensive colitis, may be helpful. Other treatments that are sometimes helpful include rectal bismuth preparations ${ }^{15}$ and arsenical (Acetarsol) suppositories, which seem to be safe and well tolerated. ${ }^{16}$ Many patients with proctitis have constipation in the proximal colon, and effective relief of this, sometimes requiring a dose of a powerful osmotic laxative such as Picolax, may help to induce a remission. There is some evidence that the diarrhoeal side effect of oral olsalazine may give it a slight advantage over other oral mesalazine preparations in proctitis for this reason. ${ }^{17}$

\section{Left sided colitis and pancolitis}

More marked diarrhoea-for example, four or more times per day-indicates that the disease extends more proximally and requires systemic corticosteroid therapy.

The optimal starting dose of oral prednisolone in an adult is $40 \mathrm{mg}$ /day. This dose is usually effective within two to four weeks, after which it should be gradually tapered off completely over about eight to 12 weeks. Delayed therapy may increase the risk of the patient requiring colectomy, so it is not appropriate to start therapy with a suboptimal dosage. There is no evidence that chronic steroid therapy is effective in maintaining remission, and significant steroid side effects are therefore usually avoided.

Once a patient has achieved remission, long term therapy should involve a standard maintenance dose of an oral 5-ASA agent. Optimal doses of mesalazine (usually $800 \mathrm{mg}$ thrice daily) or sulphasalazine ( $1 \mathrm{~g}$ twice daily) reduce the frequency of relapse by about two thirds. There is no clear evidence of any advantage of one mesalazine formulation over another with the possible exception that one study has shown a better therapeutic response with relatively high doses (necessitating nine capsules per day) of balsalazide. ${ }^{18}$

Antidiarrhoeal agents have been shown not to reduce stool frequency in patients with active ulcerative colitis, and there is anecdotal evidence that they may increase the risk of toxic megacolon.

Patients who fail to respond to oral corticosteroids within two weeks should be admitted to hospital and managed as for severe colitis (see next section). Patients who show only a partial response and who are unable to tail off steroids should be treated as for refractory ulcerative colitis (see section headed refractory ulcerative colitis).

Severe or fulminant colitis

Severe colitis is a systemic illness defined by the presence of diarrhoea accompanied by tachycardia (more than 90 beats/ min), pyrexia (more than $37.5^{\circ} \mathrm{C}$ ), a serum albumin of less than $35 \mathrm{~g} / \mathrm{l}$, or a haemoglobin of less than $100 \mathrm{~g} / \mathrm{l}$. Severe abdominal pain is also a worrying feature and, together with any of the above, merits immediate admission to hospital. Toxic megacolon (a transverse colon diameter of more than $5 \mathrm{~cm}$ ) should be excluded by a plain abdominal radiograph.

The mainstay of treatment is parenteral corticosteroid therapy, usually hydrocortisone (100 mg intravenously every 8 hours). Mesalazine is usually given in addition but is of uncertain benefit in this setting. A blood transfusion is indicated if the haemoglobin is less than $100 \mathrm{~g} / \mathrm{l}$. There is no clear evidence that bowel rest and intravenous feeding are helpful, but they may be indicated if there is evidence of malnutrition (other than hypoalbuminaemia, which is more an indication of the acute phase response in this setting).

Controlled trials have shown no benefit of the addition of intravenous metronidazole, ${ }^{19}$ but if there is any doubt over the diagnosis-for example, in a first attack or in a patient who has recently travelled in an amoebic endemic area-then it is prudent to add oral metronidazole and a quinolone such as ciprofloxacin to cover against amoebae and pathogenic bacteria, respectively.

Failure to respond-for example, persistence of fever with no diminution of diarrhoea within seven to 10 days of starting intravenous corticosteroids-is an indication for colectomy. Further prolongation of therapy will rarely avoid the need for colectomy and carries a substantial risk of perforation, which is associated with $50 \%$ mortality in this context.

Patients with toxic dilatation (megacolon) are particularly at risk of perforation and should receive colectomy within 24 hours if there is no response to therapy. ${ }^{20}$ There is some evidence that toxic megacolon can occasionally be reduced by asking the patient to assume a knees-elbow position intermittently on top of the bed. ${ }^{21}$ It should be remembered though that the likely mechanism for dilatation is smooth muscle relaxation induced by nitric oxide, which in turn is released in response to the severe inflammation, ${ }^{22}$ so this is not simply a mechanical problem.

Colectomy may also be indicated in the non-acute setting for chronic persisting colitis of lesser severity on the grounds of poor therapeutic response and poor quality of life.

The addition of ciclosporin, $4 \mathrm{mg} / \mathrm{kg}$ intravenously daily, has been shown to reduce the colectomy rate in severe colitis by about $50 \%$ in the short term and by about one third in the longer term. There is still uncertainty about whether this benefit is sufficient to justify the risks associated with the therapy, which include commensal infection, epilepsy, and nephrotoxicity. It has been suggested that the mortality associated with the use of ciclosporin of about $2 \%$ is higher than the expected mortality of less than $1 \%$ for severe colitis in a specialist centre. Blood trough ciclosporin levels should be maintained in the range of 150-300 ng/ml. Prophylaxis against Pneumocystis carinii pneumonia during therapy-for example, with cotrimoxazole-has been advised. A one week course of intravenous ciclosporin is usually followed by oral ciclosporin ( $5 \mathrm{mg} / \mathrm{kg}$ for three to six months), although there is no evidence that maintenance ciclosporin is effective in preventing relapse. ${ }^{23}$

One study has shown that a combination of a serum $\mathrm{C}$ reactive protein level exceeding $45 \mathrm{mg} / \mathrm{dl}$ and six liquid stools per day after three days of intravenous corticosteroid therapy is associated with an $85 \%$ risk of requiring colectomy. ${ }^{24}$ This is increasingly used as a means of identifying a subpopulation of patients at high risk for colectomy who might benefit from ciclosporin.

Many centres start patients who are recovering from severe colitis on maintenance azathioprine, $2 \mathrm{mg} / \mathrm{kg} /$ day (see below). 


\section{Refractory ulcerative colitis}

Azathioprine and its metabolite 6-mercaptopurine can be used interchangeably, albeit at different dosages. They are effective in $60 \%-70 \%$ of patients with refractory disease and have been shown to be beneficial in maintaining remission. ${ }^{25}$ Therapy is initiated at a dose of $50 \mathrm{mg} /$ day increasing to $1.5 \mathrm{mg} / \mathrm{kg}$ per day for 6-mercaptopurine and $2.0-2.5 \mathrm{mg} / \mathrm{kg}$ per day for azathioprine if bone marrow toxicity does not occur. A full therapeutic response to these drugs may not occur for three months. Up to $10 \%$ of patients have to stop azathioprine or 6-mercaptopurine because of acute reversible toxicities including fever, rash, pancreatitis or hepatitis. Approximately half of those patients intolerant of azathioprine, but not including those with hepatotoxicity or pancreatitis, may tolerate 6-mercaptopurine. Owing to the risk of bone marrow depression, full blood counts should be monitored after the first week, then at least monthly for three months, and once every three months thereafter, with the azathioprine or 6-mercaptopurine stopped immediately if the total white cell count falls below $3.0 \times 10^{6}$ cells $/ 100 \mathrm{ml}$. Homozygous deficiency for the enzyme thiopurine methyltransferase identifies only about one quarter of those patients who subsequently develop bone marrow suppression, ${ }^{26}$ so there is still uncertainty over whether the enzyme activity should routinely be measured before commencing therapy. There is evidence that it may be beneficial and generally safe to continue azathioprine or 6-mercaptopurine for up to five years, beyond which evidence is lacking. The theoretical possibility that immunosuppression might be associated (as in transplant recipients) with an increased risk of malignant disease has not so far been demonstrated by surveys in long term users with IBD. ${ }^{27}$

\section{Crohn's disease}

Given the heterogeneity of this disease, the options for combining therapy, and the lack of commercial interest in some of the simpler therapies, it is unlikely that sufficient controlled trials will ever be conducted to provide evidence for the best treatment for every clinical circumstance. In many situations several therapeutic options may be reasonable alternatives, and patient preference should be an important factor in determining choice of therapy. In all types of Crohn's disease cessation of smoking should be strongly encouraged. Smoking is statistically associated with Crohn's disease: two thirds of patients with Crohn's disease are smokers compared with one third of controls. Moreover, smoking is associated with higher relapse rates following surgical resection and a greater risk of perforating diseasethat is, abscess or fistula formation. ${ }^{28}{ }^{29}$ It can be argued that cessation of smoking in a patient with Crohn's disease is likely to have a more beneficial effect on the course of the disease than is any current maintenance therapy.

\section{Oral Crohn's disease}

Aphthous ulcers are the most common oral manifestation of Crohn's disease. A wide variety of additional lesions have been described, including granulomatous masses, cheilitis, and granulomatous sialadenitis. They usually co-occur with intestinal disease and respond to treatment directed at the intestinal disease. Topical therapy consisting of hydrocortisone in a carrier of pectin, gelatine, and carboxymethylcellulose or topical sucralfate may be effective in relieving local symptoms.

\section{Symptomatic gastroduodenal Crohn's disease}

Although more than $50 \%$ of patients with Crohn's disease have histological gastritis, less than $5 \%$ have symptomatic gastroduodenal disease, often in association with concurrent distal intestinal involvement. The distal antrum and varying areas of the duodenum are most commonly affected. The clinical presentation is often confused with that of peptic ulcer disease, and symptoms may include epigastric pain, nausea, and postprandial vomiting. Treatment with a proton pump inhibitor, $\mathrm{H} 2$ receptor antagonist, or sucralfate may provide partial or complete relief of symptoms. There are, however, no clinical trials in this setting. For patients with moderate to severe gastroduodenal disease prednisolone is usually effective. Azathioprine or 6-mercaptopurine is indicated in patients who remain symptomatic on steroids or who become dependent on them.

\section{Active ileal or ileocolonic disease}

The ileum is the region of the small bowel most commonly involved in Crohn's disease, and the majority of patients have either ileal or ileocolonic disease. Conventional therapy relies on oral corticosteroids and mesalazine, used in much the same way as in ulcerative colitis, but the response in patients with Crohn's disease is much less impressive than in those with ulcerative colitis. Indeed, corticosteroid therapy has been shown not to heal the ileal mucosal ulceration whereas mucosal healing has been demonstrated with enteral feeding, azathioprine, and infliximab. Antibiotics are increasingly used, although, because the nature (aerobes, anaerobes, or mycobacteria) and site (intracellular, extracellular, or gut lumen) of the target bacteria are unknown, the choice of antibiotic is highly speculative. Moreover, a lack of commercial interest has resulted in trials of antibiotics being small and often uncontrolled.

In a patient with "typical" active ileocolonic disease reasonable therapeutic options include antibiotics, azathioprine, corticosteroids, enteral nutrition, and even surgery. In a patient with short (less than $20 \mathrm{~cm}$ ) tight stricturing of the terminal ileum, resection, increasingly performed as a laparoscope-assisted right hemicolectomy, may be the most sensible initial therapy and would be expected to result in a $50 \%$ chance of remaining free of symptoms for five years, and probably better than this with appropriate postoperative medication (see later). When initial medical therapy is the preferred option the conventional choice, and the one for which most evidence is available, is corticosteroids-for example, oral prednisolone 20-60 mg/day. However, although this is likely to achieve a good symptomatic response for up to three months, it is no more effective than a placebo for longer term maintenance, it does not achieve mucosal healing, and symptomatic relapse is common when the dosage is reduced. This often results in the longer term use of corticosteroids, with concomitant side effects. Although the risk of side effects can be approximately halved by using oral budesonide (6-9 $\mathrm{mg} /$ day) in a $\mathrm{pH}$-dependent delivery system, it can be argued that there are no other conditions in which high dosages of corticosteroids are used with no expectation of affecting the natural history of the disease, and it is therefore our practice to use alternative approaches wherever possible.

An initial therapeutic response can usually be achieved with either of the antibiotics ciprofloxacin or clarithromy$\operatorname{cin}^{30-32}$ Evidence is lacking regarding optimal dosing and duration, but it is our experience that prolongation of therapy beyond three months is unhelpful.

Azathioprine (up to $2.5 \mathrm{mg} / \mathrm{kg} /$ day as for ulcerative colitis) can be used without corticosteroids. Although it has been said to take up to three months to have its full effect, this was based on incomplete short term follow up data, and azathioprine probably acts faster in most patients. In patients who cannot tolerate azathioprine, methotrexate is a good alternative. Best trial data exist for its use at a dose of $25 \mathrm{mg}$ per week (please note: this is once per week; daily administration of this dose is disastrous) by intramuscular injection. Oral methotrexate, 15-25 mg once per week, is 
often used for simplicity but is probably less effective. Oral folate, $5 \mathrm{mg} /$ day excepting the day when methotrexate is taken, is usually given to reduce the risk of side effects. Both azathioprine and methotrexate necessitate regular monitoring of full blood counts and liver function tests, initially weekly, then at intervals of one to three months. The drugs should be stopped if the total white cell count falls below $3.0 \times 10^{9}$ cells $/$, the platelet count falls below $100 \times 10^{9}$ cells $/ 1$, or liver function tests become significantly deranged-for example, an alanine transaminase of twice the upper limit of normal. Azathioprine and 6-mercaptopurine have been established to be safe during pregnancy but methotrexate is definitely contraindicated, and women of childbearing potential should be made well aware of this.

Enteral nutrition used as the sole feed is in itself an effective therapy for Crohn's disease; the mechanisms are poorly understood but probably include an effect on the intestinal bacteria. The feed can contain whole protein but there are poorly understood discrepancies between the therapeutic effects of different polymeric feeds, so a feed should be used that has been shown to be effective in Crohn's disease-for example, EO28, an amino acid based or elemental feed, or Modulen, a casein based whole protein or polymeric feed..$^{33}$ The latter can be used with Nesquick as a flavouring. Either feed is usually tolerated by mouth by about two thirds of adult patients and even better by children. In children with active Crohn's disease it is the therapy that achieves greatest height gain, and this acts as a considerable added incentive. Approximately two thirds of patients go into clinical remission with no other therapeutic intervention within three weeks of starting enteral feeding. Unfortunately, at least half of these patients relapse within six months of returning to a normal diet. We find that relapse is less common if the patient takes a low fat low fibre diet, but there is no controlled evidence to confirm this. Enteral feeding is particularly appropriate in patients with complex fistulating disease or localised intra-abdominal sepsis as a preparation (together with radiological-assisted drainage of any abscess collection) for definitive surgery. Surgery is much less likely to have a complicated outcome if the patient is well nourished and not receiving high doses of corticosteroids.

The response to mesalazine in Crohn's disease is generally disappointing; some meta-analyses have shown little more than a $5 \%$ advantage over placebo. ${ }^{35}$ However, its regular use is associated with a marked reduction in the risk of colon cancer in patients with ulcerative colitis, and, given the similar increase in the risk of cancer in patients with Crohn's disease, it is appropriate for patients with colorectal Crohn's disease to take regular mesalazine. It has also been shown to be effective in preventing postoperative recurrence (see later).

In patients with extensive small bowel disease or previous surgical resection chronic diarrhoea may be a problem even in the absence of active Crohn's disease. Loperamide is usually the initial drug of choice because of its efficacy and relative safety. Cholestyramine is an alternative for patients with non-stenosing ileitis who have chronic watery diarrhoea and is also indicated in patients with previous ileal resections who have bile salt diarrhoea.

\section{Diagnosis and management of intra-abdominal sepsis}

Patients with small bowel Crohn's disease not infrequently present with fever, chills, and localised right lower quadrant pain associated with leucocytosis. Such patients have often suffered a microperforation with localised peritonitis that is contained by the surrounding mesentery and bowel loops. Appendicitis is also a consideration in this setting. Abdominal and pelvic computed tomography is usually the most helpful diagnostic investigation. The management of localised peritonitis includes bowel rest, which may involve enteral feeding if the patient can tolerate this, and broad spectrum antibiotics. A response to therapy is usually seen within three to four days, and intravenous antibiotic therapy is continued for seven to 10 days. Intestinal resection should be considered in non-responders. However, medical management and percutaneous drainage prevented subsequent surgery in nearly half the patients in one study, suggesting that it is a reasonable approach in some patients. ${ }^{36}$

\section{Small bowel obstruction}

Small bowel obstruction is a common sequel of longstanding Crohn's disease. Conservative therapy with intravenous hydration, nasogastric suction, and parenteral nutrition is often successful, with a response seen within 24-48 hours. Surgery is reserved for patients who do not respond to these non-invasive measures.

\section{Colonic Crohn's disease}

Patients with Crohn's disease limited to the colon may present with abdominal pain, diarrhoea that is usually nonbloody, fever, and weight loss. For patients with mild to moderate symptoms, therapy can be initiated with oral mesalazine. Antibiotic therapy should be considered in patients who do not tolerate these medications or do not improve within three to four weeks. Metronidazole is the best studied of the available antibiotics, and controlled trials have supported its efficacy as a primary therapy for Crohn's ileocolitis and colitis. ${ }^{37}$ Isolated colonic Crohn's disease tends to behave more like ulcerative colitis than like other forms of Crohn's disease, and, like ulcerative colitis, it is also associated with a risk of toxic megacolon. Corticosteroidsfor example, prednisolone $40 \mathrm{mg} / \mathrm{day}$ - should therefore be used as in ulcerative colitis if there has been a poor response to mesalazine or antibiotics or both. Once remission is achieved the steroid should be tapered off completely over about three months. Immediate hospitalisation is required if patients present with severe symptoms or appear toxic.

\section{Perianal disease}

Perineal abscesses and fistulae occur in up to one third of patients with Crohn's disease. In patients who present with draining fistulae and small abscesses not amenable to surgical drainage, therapy is initiated with metronidazole at a dose of $10 \mathrm{mg} / \mathrm{kg}$ per day and increased to $20 \mathrm{mg} / \mathrm{kg}$ per day if necessary. One problem with this approach is the high rate of relapse. Long term therapy at the lowest effective dose may be necessary in such patients, but peripheral neuropathy, which is not always reversible, becomes a hazard after three months of continuous therapy, and patients should be warned to look out for symptoms of this. Ciprofloxacin can be tried in patients who do not respond to or cannot tolerate metronidazole. Azathioprine or 6-mercaptopurine should be considered in refractory patients.

\section{Fistulating Crohn's disease}

The transmural inflammatory nature of Crohn's disease predisposes to the formation of fistulae, a complication that may dominate the clinical picture. Sadly, no medical treatment has been shown to produce permanent fistula closure. Partial or temporary closure may be achieved with azathioprine or 6-mercaptopurine, enteral feeding, or infliximab. Corticosteroids do not achieve fistula closure. Patients should be managed in close partnership with a colorectal surgeon. Excision of the fistula tract may be appropriate in carefully selected cases, particularly where the fistula tract involves the small intestine or bladder. Complex perianal fistulas are rarely resectable, and aggressive surgical intervention may increase the risk of sphincter damage and incontinence. Recurrent formation of a perianal abscess can 
often be prevented by inserting a Seton suture to maintain patency of the fistula track. Infliximab is not indicated for the treatment of fistulating disease in the absence of clinically severe Crohn's disease.

\section{NEWER THERAPIES FOR REFRACTORY CROHN'S DISEASE \\ Infliximab}

Infliximab is a chimeric $\operatorname{IgG}_{1}$ monoclonal antibody comprising $75 \%$ human and $25 \%$ murine sequences, which has a high specificity for and affinity to tumour necrosis factor- $\alpha$ (TNF- $\alpha$ ). Infliximab is available for the treatment of patients with severe Crohn's disease who have had an inadequate response to conventional therapy. Treatment markedly decreases endoscopic and histological disease activity in Crohn's colitis. Preliminary results have shown that repeated therapy with infliximab is steroid sparing, especially in fistulating Crohn's disease. ${ }^{38}$ Smoking (harmful) and concomitant use of immunosuppressive drugs (helpful) have important influences on the initial response and on the durability of the response. Infliximab is contraindicated in pregnancy. Approximately 13\% of treated patients develop human antichimeric antibodies, which may increase the risk of infusion reactions to a subsequent dose. The development of these antibodies seems to be almost completely abrogated by concomitant use of azathioprine, 6-mercaptopurine, or methotrexate, and most patients should be receiving one of these agents prior to treatment with infliximab. Ongoing infections are an absolute contraindication to the use of infliximab, and MRI or computed tomography may be necessary to exclude significant sepsis before commencing infliximab. All patients who are about to receive infliximab should be screened for active or latent tuberculosis by chest radiograph. Any patient who tests positive should be treated prior to infliximab infusion.

\section{Antisense oligonucleotides}

Antisense oligonucleotides are nucleic acid sequences that bind to RNA or DNA with a high degree of specificity and can thereby block expression of a specific protein. Antisense therapy-for example, intercellular adhesion molecule- 1 and nuclear factor kappa $\mathrm{B}^{39}$-is under investigation at the experimental stage for a number of diseases including IBD.

\section{Fish oil}

A variety of proinflammatory cytokines are synthesised from n-6 fatty acids. Cytokine activity may be reduced by the administration of fish oil containing $n-3$ fatty acids. One study showed significantly higher remission with fish oil than with a placebo. ${ }^{40}$

\section{Interleukin-10}

Interleukin-10 is a cytokine that has anti-inflammatory properties and is increased in the inflamed mucosa of patients with IBD. Initial results of recombinant interleukin-10 therapy have shown rather modest benefit. ${ }^{41}$ Genetically engineered bacteria that release interleukin-10 into the gut lumen are also under investigation.

\section{Anti-integrin antibodies}

Alpha-4 integrins are involved in leucocyte migration across the vascular endothelium. An antibody against alpha-4 integrin (natalizumab) has recently been evaluated in a controlled trial of 30 patients with active Crohn's disease, with promising results. ${ }^{42}$

\section{Thalidomide}

Thalidomide decreases the production of proinflammatory cytokines, including TNF- $\alpha$ and interleukin-12. It was banned after its use in pregnancy for hyperemesis was found to cause severe birth defects. It has been shown to be effective in the treatment of Crohn's disease in open label pilot trials and may have a minor role in patients with unresponsive disease. $^{43}$

\section{Mycophenolate mofetil}

Mycophenolate mofetil is metabolised to its active moiety, mycophenolic acid, which is a non-competitive and reversible inhibitor of inosine monophosphate dehydrogenase and which has direct effects on $\mathrm{T}$ and B lymphocytes, since they require de novo synthesis of guanosine nucleotides for proliferation. Mycophenolic acid also inhibits intestinal smooth muscle growth and the synthesis of fibronectin, which might decrease smooth muscle hyperplasia and stricture formation in Crohn's disease. At best, however, it seems to have only a modest therapeutic effect. ${ }^{44}$

\section{CDP57 1}

CDP571 is a humanised monoclonal chimeric anti-TNF- $\alpha$ antibody. Its efficacy and safety were evaluated in a placebo controlled trial involving 169 patients with moderate to severe Crohn's disease. ${ }^{45}$ Whether it has any advantages over infliximab remains to be determined.

\section{Etanercept}

Etanercept is an injectable TNF receptor fusion protein that binds to and inactivates human TNF. It is currently used to treat rheumatoid arthritis. No benefit was observed in a placebo controlled four week trial in patients with Crohn's disease ${ }^{46}$ This has led to the hypothesis that infliximab acts in Crohn's disease via apoptosis of $\mathrm{T}$ lymphocytes expressing TNF, an action that is not seen with etanercept.

\section{Granulocyte macrophage colony stimulating factor}

The hypothesis that some cases of Crohn's disease may result from intramucosal phagocyte dysfunction led to a trial of recombinant granulocyte macrophage colony stimulating factor, with promising results. ${ }^{47}$

\section{Growth hormone}

Various regulatory peptides (such as growth hormone and insulin like growth factor I) are involved in the control of intestinal permeability, providing a rationale for a controlled trial of the use of growth hormone in Crohn's disease, which is associated with increased intestinal permeability, with promising results. ${ }^{48}$ More controlled studies are required before it can be recommended outside trials.

\section{Antituberculous therapy}

A number of observations have suggested a link between Crohn's disease and mycobacteria. It has been known for nearly a century that Crohn's disease resembles tuberculous gastroenteritis and is histopathologically similar to Johne's disease, an enteritis of ruminants caused by Mycobacterium paratuberculosis. Evidence for the presence of small amounts of $M$ paratuberculosis in Crohn's disease tissue is controversial. A meta-analysis of seven trials concluded that treatment directed against $M$ paratuberculosis may be helpful. ${ }^{49}$ Such treatment regimens have generally included clarithromycin and antileprotic drugs, such as clofazimine or dapsone. However, only a small number of patients have been studied. Furthermore, it is unclear whether the observed benefit is due to the treatment of mycobacteria or to a non-specific effect of the drugs, some of which have antibacterial effects on enteric flora. A larger controlled trial is currently underway.

\section{Bone marrow transplantation}

A recent small series of patients successfully treated by autologous bone marrow transplantation has been reported..$^{50}$ The rationale for this therapy is not very clear but may 
include "resetting" the immune system and reducing the immune response to the intestinal flora.

\section{SPECIAL POPULATIONS AND PRESENTATIONS Pregnancy and IBD}

Patients with quiescent IBD have the same fertility rate and risk of complications during pregnancy as the general population. ${ }^{51}$ Nutrition during the first trimester is crucial. There is a general consensus that women with active perianal Crohn's disease should have an abdominal delivery. Pregnancy does not affect the course of IBD, but undertreatment of active IBD may adversely affect the pregnancy. In active disease, medications should therefore be administered as if the patient were not pregnant (with the proviso that drugs such as methotrexate, metronidaxole and infliximab, which are contraindicated in pregnancy, should be avoided).

\section{Paediatric and adolescent IBD}

Ulcerative colitis should be treated as for an adult. A greater proportion of children than adults with Crohn's disease manifest with small bowel Crohn's disease, ${ }^{52}$ and they often respond particularly well to enteral feeding. It is extremely important to monitor growth carefully.

\section{Prevention of postoperative relapse}

Separate controlled trials have shown that regular mesalazine (Pentasa $1 \mathrm{~g}$ thrice daily) and metronidazole (400 mg thrice daily for three months) may substantially reduce the risk of postoperative relapse of Crohn's disease, particularly within the first year. Azathioprine is also being evaluated for this purpose..$^{534}$

\section{Surveillance for colorectal cancer}

Patients with ulcerative colitis or colonic Crohn's disease have an approximately fivefold increased risk of developing colorectal cancer. This is particularly marked in patients with extensive colitis. Because of this, surveillance colonoscopy is recommended for those patients who have had extensive colitis for at least eight years or more limited disease for at least 15 years. ${ }^{55}$ Colonoscopy should be performed every three years for the first ten years, then every two years for 10 years, then annually. Multiple biopsies (four from each of eight segments of the colon) are taken for pathological analysis, and colectomy is recommended if high grade dysplasia is found. This is a complex and controversial area, but the potential benefit in this group of patients is substantially

\section{Box 1: Top five papers}

- Lee SD, Cohen RD. Endoscopy in inflammatory bowel disease. Gastroenterol Clin North Am 2002;31:11932.

- Jewell DP, Caprilli R, Mortensen N, et al. Indication and timing for surgery for severe ulcerative colitis. Gastroenterol Int 1991;4:161.

- Sandborn W, Sutherland L, Pearson D, et al. Azathioprine or 6-mercaptopurine for inducing remission of Crohn's disease. Cochrane Database Syst Rev 2000;CD000545.

- Kornfield D, Cnattingius S, Ekbom A. Pregnancy outcomes in women with inflammatory bowel disease: a population based cohort study. Am J Obstet Gynecol 1997;177:942-6.

- Podolsky DK. Medical progress: inflammatory bowel disease. N Engl J Med 2002;347:417-29.

\section{Box 2: Top five websites}

- www.uptodate.com

- www.bsg.org.uk

- www.nacc.org.uk

- www.ccfa.org

- www.cochrane.org

greater than that in the normal population, particularly because of the relatively young age (median of 55 years) at which colorectal cancers occur in patients with colitis. ${ }^{56}$ Although there are no controlled data showing that surveillance colonoscopy reduces overall mortality, a casecontrol study has shown a strong trend towards reduced mortality, which is associated with a "dose response" - that is, a greater reduction in mortality in those who had undergone more frequent colonoscopy. ${ }^{57}$ Patients undergoing such surveillance should be reassured that the overall life expectancy of patients with ulcerative colitis is normal; any increased risk of colon cancer is balanced by a reduced risk of cardiovascular disease..$^{58}$

\section{Authors' affiliations}

M Nayar, J M Rhodes, Department of Medicine, University of Liverpool, Liverpool, UK

\section{REFERENCES}

1 Jenkins D, Balsitis M, Gallivan S, et al. Guidelines for the initial biopsy diagnosis of suspected chronic idiopathic inflammatory bowel disease. London: British Society of Gastroenterology, 1997.

2 Scott EM, Gaywood I, Scott BB. Guidelines for osteoporosis in celiac disease and inflammatory bowel disease. Gut 2000;46(suppl 1):i1-i8.

3 British Society of Gastroenterology. Inflammatory bowel disease. London: British Society of Gastroenterology, 1996.

4 Gore RM, Laufer I. Ulcerative and granulomatous colitis: idiopathic inflammatory bowel disease. In: Gore RM, Levine MS, Laufer I, eds. Textbook of gastrointestinal radiology. Philadelphia: WB Saunders, 1994:1098-141.

5 Scotiniotis I, Rubesin SE, Ginsberg GG. Imaging modalities in inflammatory bowel disease. Gastroenterol Clin North Am 1999;28:391-421.

6 Balthazar EJ. CT of the gastrointestinal tract: principles and interpretation. Am J Roentgenol 1991;156:23-32.

7 Lee SD, Cohen RD. Endoscopy in inflammatory bowel disease. Gastroenterol Clin North Am 2002;31:119-32.

8 Parente $F$, Cucino C, Bollami S, et al. Focal gastric inflammatory infiltrates in inflammatory bowel disease: prevalence, immunohistochemical characteristics and diagnostic role. Am J Gastroenterol 2000;95:705-11.

9 Riddel RH. Pathology of inflammatory bowel disease. In: Kirsner JB, ed. Inflammatory bowel disease. Philadelphia: WB Saunders, 2000:427.

10 Quinton JF, Sendid B, Reumaux D, et al. Anti Saccharomyces cerevesiae mannan antibodies combined with antineutrophil cytoplasmic autoantibodies in inflammatory bowel disease: prevalence and diagnostic role. Gut 1998;42:788-91

11 Moore BA, Otterbein LE, Turler A, et al. Inhaled carbon monoxide suppresses the development of postoperative ileus in the murine small intestine. Gastroenterology 2003;124:377-91

12 Marteau P, Crand J, Foucault M, et al. Use of mesalazine slow release suppositories $1 \mathrm{~g}$ three times per week to maintain remission of ulcerative proctitis: a randomised double blind placebo controlled multicentre study. Gut 1998:42:195-9.

13 Hanauer SB, Robinson M, Pruitt R, et al. Budesonide enema for the treatment of active, distal ulcerative colitis and proctitis: a dose-ranging study. Gastroenterology 1998;115:525-32.

14 Marshall JK, Irvine EJ. Rectal corticosteroids versus alternative treatments in ulcerative colitis: a meta-analysis. Gut 1997;40:775-81.

15 Ryder SP, Walker RJ, Rhodes JM, et al. Rectal bismuth subsalicylate therapy for ulcerative colitis. Aliment Pharmacol Ther 1990:4:333-8.

16 Forbes A, Britton TC, Gazzard BG. Safety and efficacy of acetarsol suppositories in unresponsive proctitis. Aliment Pharmacol Ther 1989;3:553-6.

17 Zinberg J, Molinas S, Das KM. Double-blind placebo-controlled study of olsalazine in the treatment of ulcerative colitis. Am J Gastroenterol 1990;85:562-6.

18 Levine DS, Riff DS, Johnson LK, et al. Randomised double blind dose response comparison of balsalazide $(6.75 \mathrm{~g})$, balsalazide $(2.25 \mathrm{~g})$ and mesalamine $(2.4 \mathrm{~g})$ in the treatment of active, mild-to-moderate ulcerative colitis. Am J Gastroenterol 2002:97:1398-407. 
19 Chapman RW, Selby WS, Jewell DP. Controlled trial of intravenous metronidazole as an adjunct to corticosteroids in severe ulcerative colitis. Gut 1986;27:1210-12.

20 Jewell DP, Caprilli R, Mortensen N, et al. Indication and timing for surgery for severe ulcerative colitis. Gastroenterol Int 1991;4:161-4.

21 Panos MZ, Wood MJ, Asquith P. Toxic megacolon: the knee-elbow position relieves bowel distension. Gut 1993;34:1726-7.

22 Guslandi M. Nitric oxide in inflammatory bowel disease. Eur J Clin Invest 1998;28:904-7.

23 Kornbluth A, Present DH, Lichtiger S, et al. Cyclosporine for severe ulcerative colitis: a user's guide. Am J Gastroenterol 1997;92:1424-8.

24 Travis SP, Farrant JM, Ricketts C, et al. Predicting outcome in severe ulcerative colitis. Gut 1996;38:905-10.

25 Present DH, Chapman ML, Rubin PH, ef al. Efficacy of 6-MP in refractory ulcerative colitis. Gastroenterology 1988;94:359A.

26 Colombel JF, Ferrari N, Debuysere H, et al. Genotypic analysis of thiopurine S-methyltransferase in patients with Crohn's disease and severe myelosuppression during azathioprine therapy. Gastroenterology 2000; 118:1025-30.

27 Connell WR, Kamm MA, Dickson M, et al. Long-term neoplasia risk after azathioprine treatment in inflammatory bowel disease. Lancet 1994;343:1249-52.

28 Cosnes J, Beaugerie L, Carbonnel F, et al. Smoking cessation and the course of Crohn's disease: an intervention study. Gastroenterology $2001 ; 120: 1093-9$

29 Louis E, Michel V, Hugot JP, et al. Early development of stricturing or penetrating pattern in Crohn's disease is influenced by disease location, number of flares, and smoking but not by NOD2/CARD15 genotype. Gut 2003;52:552-7.

30 Prantera C, Zannoni F, Scribano ML, et al. An antibiotic regime for the treatment of active Crohn's disease: a randomised controlled clinical trial of metronidazole plus ciprofloxacin. Am J Gastroenterol 1996;91:328-32.

31 Moss AA, Carbone JV, Kressel HY. Radiologic and clinical assessment of broad-spectrum antibiotic therapy in Crohn's disease. Am J Roentgenol 1978;131:787-90.

32 Leiper K, Morris Al, Rhodes JM. Open label trial of oral clarithromycin in active Crohn's disease. Aliment Pharmacol Ther 2000;14:801-6.

33 Verma S, Brown S, Kirkwood B, et al. Polymeric versus elemental diet as primary treatment in active Crohn's disease: a randomised double blind trial. Am J Gastroenterol 2000;95:735-9.

34 Fell JM, Paintin M, Arnaud-Battandier F, et al. Mucosal healing and a fall in mucosal pro-inflammatory cytokine mRNA induced by a specific oral polymeric diet in paediatric Crohn's disease. Aliment Pharmacol Ther 2000;14:281-9.

35 Messori A, Brignola C, Trallor G, et al. Effectiveness of 5-aminosalicylic acid for maintaining remission in patients with Crohn's disease: a meta-analysis. Am J Gastroenterol 1994;89:692-8.

36 Garcia JC, Persky SE, Bonis PA, et al. Abscesses in Crohn's disease: outcome of medical versus surgical treatment. J Clin Gastroenterol 2001;32:409-12.

37 Ursing B, Alm T, Barany $F$, et al. A comparative study of metronidazole and sulfasalazine for active Crohn's disease: the cooperative Crohn's disease study in Sweden. II. Result. Gastroenterology 1982;83:550-62.

38 Present DH, Rutgeerts P, Targan S, et al. Infliximab for the treatment of fistulas in patients with Crohn's disease. N Engl J Med 1999;340:1398-405.

39 Yacyshyn BR, Chey WY, Goff J, et al. Double blind, placebo controlled trial of the remission inducing and steroid sparing properties of an ICAM-1 antisense oligodeoxynucleotide, alicaforsen (ISIS 2302), in active steroid dependent Crohn's disease. Gut 2002;51:30-6.

40 Belluzzi A, Brignola C, Campieri M, et al. Effect of an enteric-coated fish oil preparation on relapses in Crohn's disease. N Engl J Med 1996:334:1557-60.

41 Schreiber S, Fedorak RN, Nielsen $\mathrm{OH}$, et al. Safety and efficacy of recombinant human interleukin 10 in chronic active Crohn's disease. Crohn's disease IL-10 cooperative study group. Gastroenterology 2000:119:1461-72.

42 Ghosh S, Goldin E, Gordon FH, et al. Natalizumab for active Crohn's disease: the natalizumab pan European study group. N Engl J Med 2003;348:24-32

43 Vasiliauskas EA, Kam LY, Abreu-Martin MT, et al. An open-label pilot study of low-dose thalidomide in chronically active, steroid-dependent Crohn's disease. Gastroenterology 1999;117:1278-87.

44 Hassard PV, Vasiliauskas EA, Kam LY, et al. Efficacy of mycophenolate mofetil in patients failing 6-mercaptopurine or azathioprine therapy for Crohn's disease. Inflamm Bowel Dis 2000;6:16-20.

45 Sandborn WJ, Feagan BG, Hanauer SB, et al. An engineered human antibody to TNF (CDP571) for active Crohn's disease: a randomized doubleblind placebo-controlled trial. Gastroenterology 2001;120:1330-8.

46 Sandborn WJ, Hanaver SB, Katz S, et al. Etanercept for active Crohn's disease: a randomized, double-blind, placebo-controlled trial. Gastroenterology 2001;121:1088-94.

47 Dieckgraefe BK, Korzenik JR. Treatment of active Crohn's disease with recombinant human granulocyte-macrophage colony-stimulating factor. Lancet 2002;360:1478-80.

48 Slonim AE, Bulone L, Damore MB, et al. A preliminary study of growth hormone therapy for Crohn's disease. N Engl J Med 2000;342:1633-7.

49 Borgaonkar M, Maclntosh D, Fardy J, et al. Anti-tuberculous therapy for maintaining remission of Crohn's disease. Cochrane Database Syst Rev 2000;(2):CD000299.

50 Burt RK, Traynor A, Oyama Y, et al. High-dose immune suppression and autologous hematopoietic stem cell transplantation in refractory Crohn disease. Blood 2003;101:2064-6.

51 Kornfeld D, Cnattingius S, Ekbom A. Pregnancy outcomes in women with inflammatory bowel disease: a population based cohort study. Am J Obstet Gynecol 1997; 177:942-6.

52 Cuffari C, Bayless TM. Crohn's disease: influence of age at onset on clinical phenotype. Gastroenterol Int 1997; 10:89-98.

53 Leiper K, London I, Rhodes JM. Adjuvant post operative therapy. Bailleres Clin Gastroenterol 1998;12:179-99.

54 Caprilli R, Cottone $M$, Tonelli $F$, et al. Two mesalamine regimens in the prevention of the post operative recurrence of Crohn's disease: a pragmatic double blind randomised control trial. Aliment Pharmacol Ther 2003;17:517-23.

55 Eaden JA, Mayberry JF. Guidelines for screening and surveillance of asymptomatic colorectal cancer in patients with inflammatory bowel disease. Gut 2002;51(suppl V):v10-v12.

56 Rhodes JM. Surveillance for colitis-associated cancer: we cannot stop now. Dig Liver Dis 2002;34:319-21.

57 Karlen $\mathbf{P}$, Kornfeld $\mathrm{D}$, Brostrom $\mathrm{O}$, et al. Is colonoscopic surveillance reducing colorectal cancer mortality in ulcerative colitis? A population based case control study. Gut 1998;42:711-14.

58 Travis SP. Review article: insurance risks for patients with ulcerative colitis or Crohn's disease. Aliment Pharmacol Ther 1997;1 1:51-9. 